\title{
Enterprise Data Backup \& Recovery: A Generic Approach
}

\author{
Majid Zaman, Muheet Ahmed Butt \\ Scientist, Directorate of IT \& SS, University of Kashmir, Srinagar, India \\ Scientist, PG Department of Computer Science, University of Kashmir, India
}

\begin{abstract}
Even with the onset of 21st century there is no single backup and recovery tool available which can be used irrespective of type of Database Management System and the platform on which it is functioning. Enterprises are now days using n (multiple) heterogeneous data sources of varying Database Management Systems located at geographically distant locations interconnected by internet (intranet) and as such have to manage $\mathrm{n}$ backups which in turn requires $\mathrm{n}$ backup and recovery systems. While ORACLE dominates the market in large enterprises, yet its backup and recovery tool cannot be used for the backup and recovery of data stored on MYSQL Database Management System and/or MSSQL Database Management System. Enterprises have to pay hefty sum on backup and recovery based on the number of licenses the enterprise has acquired. In this paper we propose a backup and recovery solution which makes use of the existing database architectures and takes data backup in a manner irrespective of any specific Database Management System which can be used to recover any Database System.
\end{abstract}

\section{INTRODUCTION}

As data is the heart of the enterprise, it becomes crucial as well as important for us to protect it. And to protect our organization's data, we need to implement a data backup and recovery plan. Backing up files can protect against accidental loss of user data, database corruption, hardware failures, and even natural disasters. It's our job as an administrator to make sure that backups are performed and are stored in a secure location. In information technology, a backup, or the process of backing up, refers to the copying and archiving of computer data so it may be used to restore the original after a data loss event. Backups have two distinct purposes. The primary purpose is to recover data after its loss, be it by data deletion or corruption. The secondary purpose of backups is to recover data from an earlier time, according to a user-defined data retention policy, typically configured within a backup application for how long copies of data are required. Though backups popularly represent a simple form of disaster recovery, and should be part of a disaster recovery plan, by themselves, backups should not alone be considered disaster recovery. One reason for this is that not all backup systems or backup applications are able to reconstitute a computer system or other complex configurations such as a computer cluster, active directory servers, or a database server, by restoring only data from a backup. Since a backup system contains at least one copy of all data worth saving, the data storage requirements can be significant. Organizing this storage space and managing the backup process can be complicated undertaking. A data repository model can be used to provide structure to the storage. Nowadays, there are many different types of data storage devices that are useful for making

\section{PROBLEM STATEMENT}

In order to have generic solution for database backup and recovery it should essentially have following features

\section{Backup of}

i. Entire DBMS, which includes all the databases, tables existing in DBMS.

ii. Specific Database within Database Management System.

iii. Specific Tables within Database of Database Management System.

The administrator should have the ability to take backup of any one of the above, irrespective of the type of Database Management System.

\section{Recovery of}

Recovery should be possible on any desired server e.g. backup taken of MySQL table on Linux server can be recovered on MySQL Database Server installed on windows server. Recovery should be possible for all the above mentioned possibilities.

\section{PROPOSED SOLUTION}

Users observed that backup is important as far as data is considered. They expressed frustration at everincreasing costs for solutions that no longer do what they're supposed to do because they are overwhelmed 
by data growth rates and the impact of server virtualization. Backups are not getting done on time. Recovery takes too long. Users are driven to multiple solutions to solve specific problems. Recovery is complex and unreliable. Costs are continually rising. What's needed is a single, integrated combination of hardware and software that resolves all of these problems while delivering ease of use and cost savings from end to end. This solution will involve in implementation of database backup techniques. We start our solution by implement the following goals. That solution is proposed below.

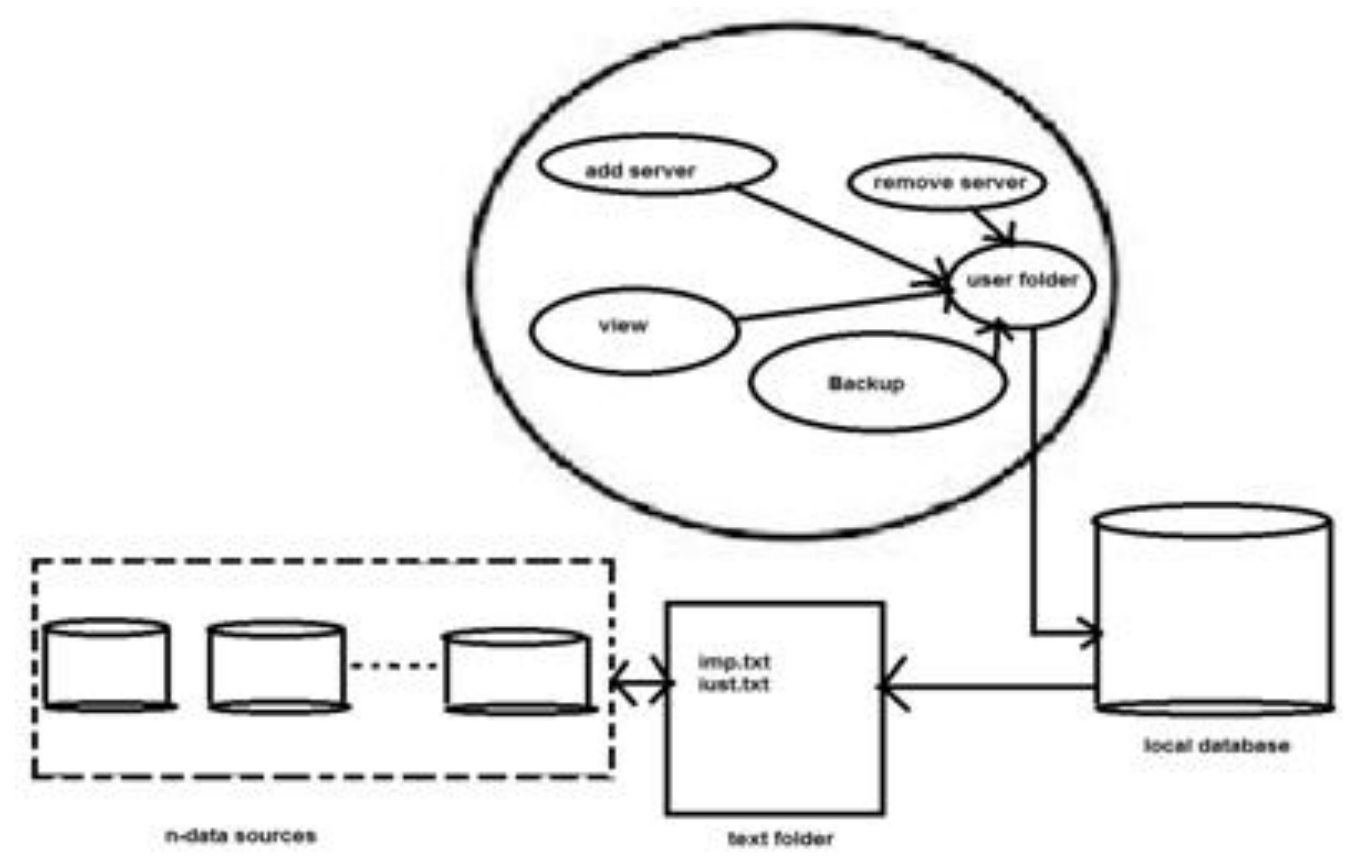

Figure 1 Retrieving Data from n Data Sources

\subsection{Identification of server based on}
$\checkmark$ IP Address.
$\checkmark$ User Name \&
$\checkmark$ Password.

\subsection{In order to take Backup}

$\checkmark$ Added servers are accessed, based on information like its ip address, username and password.

$\checkmark$ Software does not manipulate i.e. update delete any data present on client's database, it only fetches data from the said servers.

$\checkmark$ Fetched data is converted into queries and saved into test files which is generated for storage of queries.

$\checkmark$ Data is viewed, retrieved and converted into the query and then saved into text file. This text file is then saved onto the server. If required multiple backups of same database/tables can be taken.

\subsection{In order to Restore Backup}

$\checkmark$ Servers are identified, based on ip address, username and password.

$\checkmark$ User selects the backup to be restored on the server identified

$\checkmark$ Selected backup which is text file is read query by query \& said queries are executed on the identified server one by one, these queries may include table definition queries also.

$\checkmark$ Same backup can be restored on multiple servers irrespective of type of Database Management System.

\subsection{Procedure (Operation of System)}

$\checkmark$ Authenticated user logs in using an authentic username and password.

$\checkmark$ User adds its server, which is a data source.

$\checkmark$ Authenticate server using an ip address, user name, and password.

$\checkmark$ For taking backup of data, Take_backup button is clicked.

$\checkmark$ One among the two buttons (Database or Table) is clicked depending on what user needs to take backup of.

$\checkmark$ Desired database or table is selected and Take_backup button is clicked.

$\checkmark \quad$ A backup file (text file) is created. 


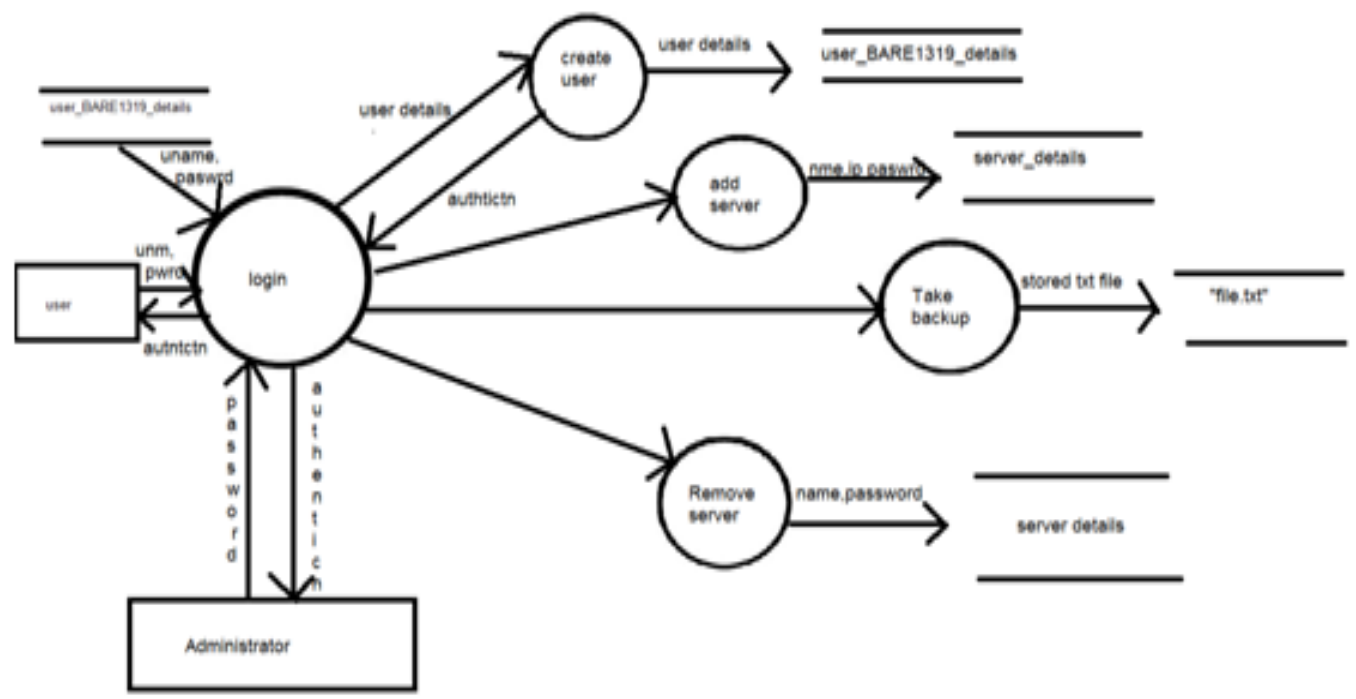

Figure 2. Proposed Backup Process

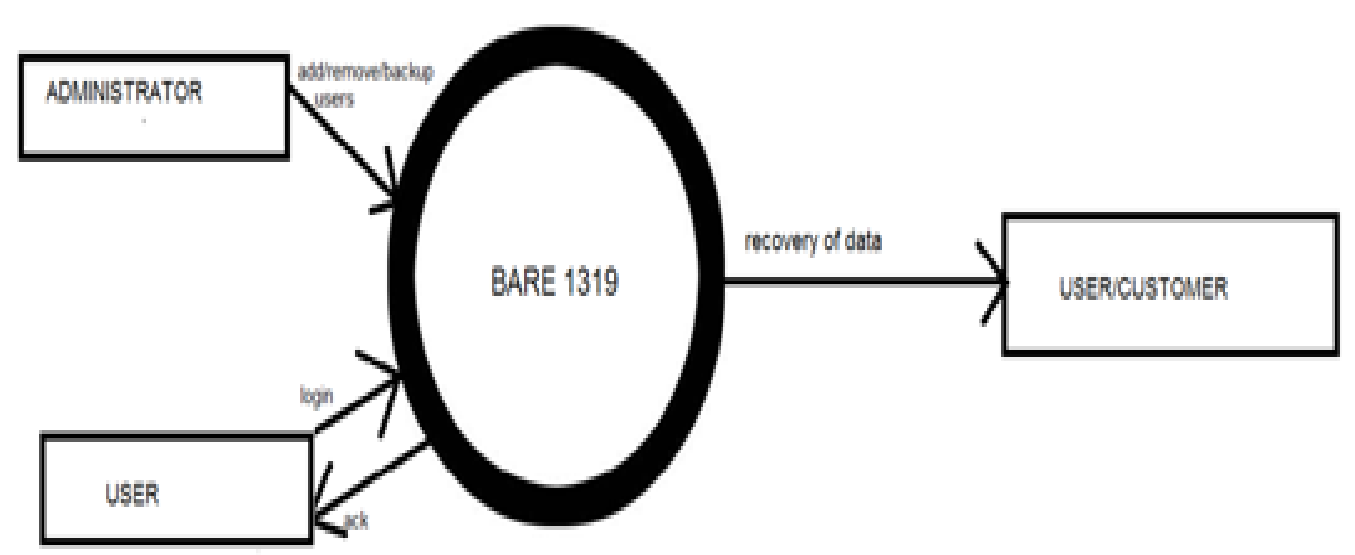

Figure 3: Proposed Recovery Process

\section{CONCLUSION}

To conclude the backup and recovery refers to the various strategies and procedures involved in protecting your database against data loss and reconstructing the database after any kind of data loss. Various suitable measures are adopted for implementing the backup and recovery process. The proposed research provides a suitable way of implementing backup and restores operations which can be implemented for enterprise. These backup and restore solutions are simple easy to implement and could be employed to any single or multiple database systems.

\section{REFERENCES}

[1]. M Zaman, MA Butt, DSMK Quadri, “User Desired Information Translation”Journal of Global Research in Computer Science 3 (6), 51-53

[2]. EMA Butt, SMK Quadri, EM Zaman, "Star Schema Implementation for Automation of Examination Records", WORLDCOMP, 2012 FEC3568, Las Vegas USA.

[3]. MA Butt, SMK Quadri, M Zaman," Data Warehouse Implementation of Examination Databases", International Journal of Computer Applications 44 (5), 18-23.

[4]. Buhalis, D \& Laws, E. (eds) (2001). Tourism Distribution Channels-Practices, Issues and Transformations, Continuum Publishing, London.

[5]. Buhalis, D. (2003). e tourism -Information Technology for strategic tourism management, Prentice Hall, Harlow, UK.

[6]. Carter R \& Bedard, F. (2001). E-Business for Tourism-Practical Guidelines for Tourism Destinations and Business, WTO Business Council, Madrid.

[7]. Carter R \& Richer P. (1999). Marketing Tourism Destination Online, WTO Business Council, Madrid. 